\title{
Infective esophagitis: inhaled steroids treatment for eosinophilic esophagitis not always safe
}

\begin{abstract}
Steroids, inhaled and swallowed, are widely used for the management of asthma and eosinophilic esophagitis (EoE). Oro-pharyngeal infections are well-recognised complications. Whilst esophagitis secondary to Herpes simplex virus (HSV) and cytomegalovirus (CMV) are a well-defined entity in patients on long-term inhaled steroids, this is likely an under-diagnosed condition as few clinical reports are available in the literature in immune-competent patients. Here, we report two unusual cases of HSV esophagitis and CMV esophagitis in a 23-year-old male and a 57-year-old female respectively. Both patients were immune-competent and were on long term inhaled steroid therapy for EoE.
\end{abstract}

Keywords: eosinophilic esophagitis, inhaled steroids, herpes simplex virus, cytomegalovirus
Volume 6 Issue 4 - 2017

\author{
Alkesh V Zala, ${ }^{1,2,3}$ Lucas Wauters, ${ }^{4}$ Tim \\ Vanuytsel, ${ }^{4}$ Steven Bollipo, ${ }^{1,2,3}$ Jan Tack, ${ }^{4}$ \\ Marjorie MWalker, ${ }^{2,3,5}$ Nicholas J Talley ${ }^{1,2,3}$ \\ 'Department of Gastroenterology, John Hunter Hospital, \\ Australia \\ ${ }^{2} S$ chool of Medicine and Public Health, University of Newcastle, \\ Australia \\ ${ }^{3}$ Australian Gastrointestinal Research Alliance Group, Hunter \\ Medical Research Institute, Australia \\ ${ }^{4}$ Department of Gastroenterology and Hepatology, University \\ Hospitals Leuven, Belgium \\ ${ }^{5}$ Department of Anatomical Pathology, Pathology North, John \\ Hunter Hospital, Australia
}

Correspondence: Alkesh Zala, Gastroenterology Department, John Hunter Hospital, Newcastle, Australia, Tel 6I 430092752 Fax 6I2432I7788, Email alkesh.zala@hnehealth.nsw.gov.au

Received: January 04, 2017 | Published: March 14, 2017

Abbreviations: CMV, cytomegalovirus; CRP, c-reactive protein; EGD, esophagogastroscopy; EoE, eosinophilic esophagitis; HIV, human immunodeficiency virus; HSV, herpes simplex virus; PPI, proton pump inhibitor; WBC, white blood cell

\section{Introduction}

Eosinophilic esophagitis (EoE) is a chronic immune-mediated disease, clinically defined by oesophageal symptoms and pathologically confirmed by eosinophilic cell infiltration. Treatment with inhaled steroids is commonly prescribed but infectious complications of steroid treatment have not been frequently reported. We describe two cases of complicated treatment of EoE with Herpes simplex (HSV) and cytomegalovirus (CMV) in immune-competent patients.

\section{Case description}

\section{Case I}

A 23-year-old male presented with acute dysphagia and odynophagia for solids. He had multiple episodes of vomiting, retrosternal chest pain and fever but denied shortness of breath of cough. He was previously diagnosed with EoE aged 16years following presentation with an acute food bolus obstruction, on a background of long standing dysphagia. His other medical history was unremarkable, with no history of allergies or atopy. He had no known history of HSV infection. Following the initial diagnosis, he was treated with inhaled Fluticasone $250 \mathrm{mcg} 2$ puffs twice a day swallowed and Omeprazole $20 \mathrm{mg}$ daily with symptomatic relief. The repeat esophagogastroscopy (EGD) showed some persistent ridging and edema. Biopsy showed showed persistent features of EoE with raised eosinophil counts. Overall, he had symptomatic improvement but EoE remained resistant to standard treatment endoscopically and histologically.
On current presentation, the white blood cell (WBC) count were $11.2 \times 10^{9} / \mathrm{L}$ and C-reactive protein (CRP) was $115 \mathrm{mg} / \mathrm{L}$. An urgent EGD was performed. The EGD revealed severe esophagitis with ulceration which was biopsied and sent for histology (Figure 1).
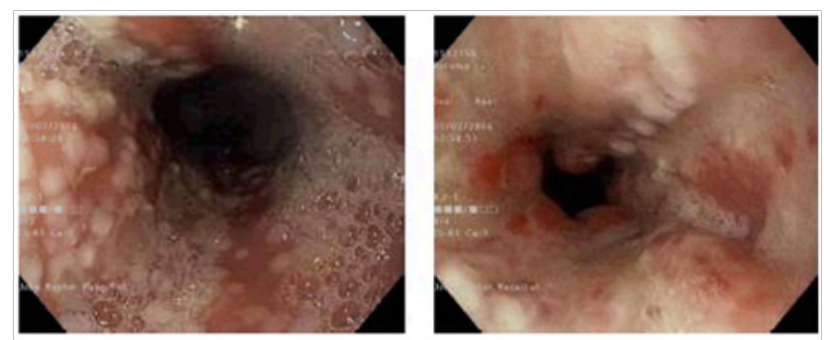

Figure I Severe esophagitis and ulceration at EGD.

Based on the initial provisional EGD diagnosis of esophagitis secondary to Candida or HSV, he was treated with fluconazole, acyclovir and pantoprazole. The biopsy results confirmed HSV infection by immunocytochemistry, and fluconazole was ceased (Figure 2). He continued on acyclovir with full resolution of symptoms. Screening tests for immune-suppression, including Human Immunodeficiency virus (HIV) were negative. The steroid aerosol was discontinued and he was offered a follow-up EGD after a period of observation. There was no recurrence of dysphagia despite no treatment. At follow up EGD, there were no endoscopic features of EoE although the oesophageal biopsies confirmed EoE and follow up continues.

\section{Case 2}

A 57-year-old female presents with a history of intermittent odynoand dysphagia for both liquids and solids, with no response to oral proton pump inhibitor (PPI) therapy. She was diagnosed with EoE 
at the age of 56 after investigation for dysphagia. She was started on inhaled Fluticasone $250 \mathrm{mcg}$ swallowed and Budesonide $3 \mathrm{mg}$ daily, with symptomatic benefit. The treatment was complicated by recurrent oesophageal candidiasis and treated with Fluconazole 100mg once a day, with resolution of odynophagia but persisting dysphagia.

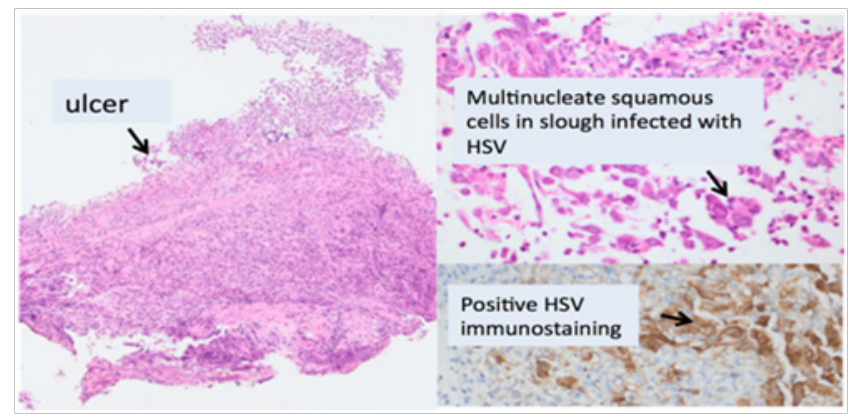

Figure 2 Histology shows ulcerated oesophageal squamous mucosa with multinucleate cells in slough, which stain positively with HSV immunostaining.

On current presentation, the total WBC count was normal. EGD showed sclerotic appearance of the entire oesophagus, with distal verrucous ulcerating mucosa (Figure 3). Pathologic examination of the distal biopsies reported columnar-lined epithelium and large stromal mesenchymal cells with eosinophilic intranuclear inclusions, surrounded by a lympho-plasmacytic and neutrophilic cell infiltrate and immunoreactive to CMV staining (Figure 4). Blood CMV polymerase chain reaction assay was positive.

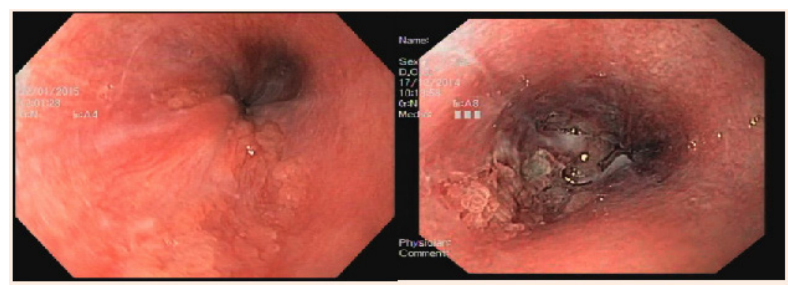

Figure 3 Post-operative MRI lumbo-sacral region, shows adequate drainage of pus with fixation.

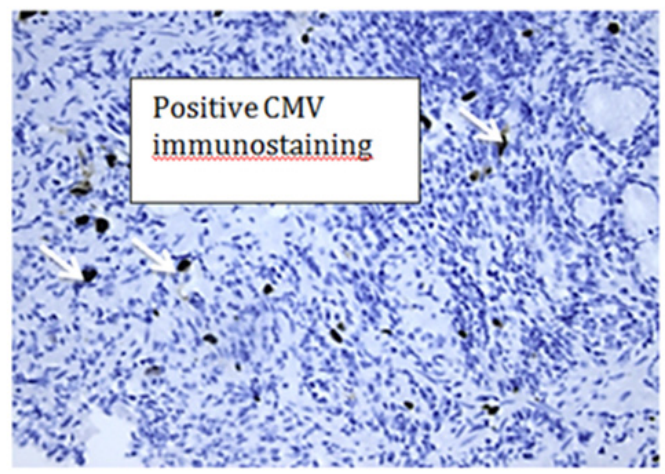

Figure 4 Histology shows characteristic intranuclear inclusions (arrowed), which stain positively with CMV immunostaining.

The diagnosis of CMV esophagitis was made and the patient was treated with Ganciclovir intravenously for 2 weeks. Tests for underlying immunodeficiency and HIV were negative. At follow-up EGD, there was a sclerotic oesophageal lumen. The CMV, HSV and fungal staining were however negative. After consulting a dietician, a 6-food elimination diet was proposed with little effect. Budesonide oral gel was trialled and the patient remains in follow-up.

\section{Discussion}

Eosinophilic esophagitis is an immune-mediated disease affecting the esophagus with increasing recognition and prevalence. ${ }^{1}$ It is characterized histologically by eosinophil-predominant inflammation of the esophagus. ${ }^{2}$ The exact pathogenesis of the disease is unknown but it is closely associated with atopic disease such as asthma, allergic rhinitis and eczema. ${ }^{3}$ A complex interplay of environmental/ food allergen and host immune system in a genetically predisposed individual forms the basis. ${ }^{4,5}$ It presents with a variety of esophageal symptoms, including dysphagia, food impaction, atypical chest pain and heartburn. ${ }^{6}$ The constellation of symptoms and typical endoscopic and histological findings are diagnostic. The endoscopic findings include mucosal friability, ringed appearance, longitudinal furrows, white papules and stricture or narrowed lumen. ${ }^{6}$ The American College of Gastroenterology recommends a cut of $>15$ eosinophils per high power field on the esophageal biopsy in presence of esophageal symptoms that are not responsive to proton pump inhibitors for diagnosis. ${ }^{7}$ Very little is known over the natural history of the disease, but current evidence suggests a favorable prognosis. ${ }^{8}$

\section{Treatment of EoE}

The available treatment options include elemental or elimination diets, proton pump inhibitors to treat gastro-esophageal reflux disease that may mimic eosinophilic esophagitis, topical glucocorticoids, systemic glucocorticoids and esophageal dilation to treat strictures.

A strict elemental diet can lead to complete resolution of the symptoms but symptoms recur on resumption of normal diet. However, often patients find them unpalatable and adherence is poor. ${ }^{9}$ Similarly, 6 foods (egg, wheat, soy, cow's milk protein, seafood, peanuts) elimination diet has shown beneficial results but large scale randomized trails are lacking. ${ }^{10,11}$ Acid suppression with proton pump inhibitors still has an unclear role but they often lead to improvement. However, current guidelines recommend them especially in patients with reflux symptoms. ${ }^{12}$ Considering its effectiveness and lack of systemic side effects, both swallowed fluticasone propionate administered via a metered dose inhaler and oral viscous budesonide have been used and shown to be effective for both symptomatic and histological improvement. ${ }^{13,14}$

There is limited data on the optimum dosage and duration of the inhaled steroid therapy. A recent randomized trial evaluated the efficacy and safety of high-dose swallowed fluticasone and dose reduction in patients with EoE. At least 3months of Fluticasone induced histologic remission in $65 \%-77 \%$ of patients and $50 \%$ dose reduction remained effective in $73 \%-93 \%$ of patients who initially responded to it. ${ }^{15}$ This suggests that all the patients should have dose reduction following a repeat endoscopy documenting histological response at the end of 3 months and then continued on lowest effective dose with appropriate follow up. Due to relapsing and remitting nature of the disease, it often recurs after the discontinuation of treatment. ${ }^{16}$ Therefore, as in our case, many patients will require long-term maintenance therapy with potential higher risk of side effects.

\section{Infective esophagitis}

Infective esophagitis due to candida, HSV and CMV occurs more frequently in immune-compromised hosts. Co-infection is common. ${ }^{17}$ Esophageal candidiasis and herpes esophagitis due to the immunosuppressive effects of swallowed fluticasone is well reported in the literature. ${ }^{18-20}$ However, to our knowledge, CMV esophagitis associated with inhaled fluticasone has not been reported so far. The 
characteristic endoscopy and biopsy findings in infective esophagitis differs according to the causative agents. Candida esophagitis is seen as white mucosal plaque-like lesions on endoscopy and distinct yeast and pseudohyphae with invasion of mucosal cells on histology. There are Vesicles or ulcers with frequently normal intervening mucosa in HSV esophagitis. The Ulcers are $<2 \mathrm{cms}$, well circumscribed and have a "volcano-like" crater. Erosion, plaques and exudates are also seen. Histology shows multinucleated giant cells with ground-glass nuclei and eosinophilic inclusions (Cowdry type A inclusion bodies). Endoscopy in CMV esophagitis shows well-demarcated large linear and deep ulcer with or without pseudo membrane formation. Intranuclear or intra-Cytoplasmic viral inclusions, typical owls eye Cowdry inclusions are seen on histology. ${ }^{17,21}$

\section{Treatment of infective esophagitis}

\section{HSV esophagitis}

Whilst spontaneous resolution within one to two weeks is reported in immunocompetent patients, a short course of 7 to 10days' treatment with nucleoside analogues such as acyclovir (400mg five times a day), famciclovir (500mg three times daily) or valacyclovir (1gram three times daily) is recommended. Immune-compromised patients should receive a longer duration of therapy. There are no randomised trials comparing nucleoside analogues in HSV esophagitis. However, case reports support therapeutic benefit in hastening resolution of lesions. ${ }^{22}$ Patients with non resolution of symptoms by 5 to 7 days on acyclovir may have a virus with thymidine kinase mutations. Antiviral agents acting on DNA polymarase such as foscarnet is recommended in these patients. $^{23}$

\section{CMV esophagitis}

Ganciclovir or valganciclovir are the mainstay of treatment. The other second-line options include foscarnet, maribavir or leflunomide. Oral valganciclovir is as effective as intravenous ganciclovir when used as an initial treatment. Foscarnet has shown equal efficacy as ganciclovir in a randomized trial. ${ }^{24}$ The duration of initial induction therapy is 3 to 6 weeks. However, the duration of treatment should be based on the response of the patient. Chronic maintenance treatment is recommended if there is co-existent retinitis or recurrent disease.

\section{Conclusion}

Whilst esophagitis secondary to herpes simplex is a defined entity in healthy patients on long-term inhaled steroids, this is likely an under diagnosed condition as few clinical reports are available in the literature. CMV esophagitis as a complication of inhaled steroids in an immune-competent host has not been previously reported. Patients treated with high doses of inhaled corticosterids are at higher risk of developing infective esophagitis. Endoscopic evaluation should be offered to these patients if there are any suspicious symptoms. Although patients usually respond well to treatment without any systemic complications, it is important for health care professionals to be aware of these important complications in patients on long term inhaled steroids, particularly those with EoE. Histology is characteristic, and it is helpful to provide full clinical information of a suspicion of this condition in the histopathology request for prompt immunocytochemical staining to confirm the diagnosis.

\section{Acknowledgments}

None.

\section{Conflicts of interest}

Author declares there are no conflicts of interest.

\section{Funding}

None.

\section{References}

1. Nonevski IT, Downs-Kelly E, Falk GW. Eosinophilic esophagitis: an increasingly recognized cause of dysphagia, food impaction, and refractory heartburn. Cleve Clin J Med. 2008;75(9):623-633.

2. Liacouras CA, Furuta GT, Hirano I, et al. Eosinophilic esophagitis: updated consensus recommendations for children and adults. $J$ Allergy Clin Immunol. 2011;128(1):3-20.

3. Roy-Ghanta S, Larosa DF, Katzka DA. Atopic characteristics of adult patients with eosinophilic esophagitis. Clin Gastroenterol Hepatol. 2008;6(5):531-535.

4. Cello JP. Eosinophilic gastroenteritis--a complex disease entity. Am J Med. 1979;67(6):1097-1104.

5. Noel RJ, Putnam PE, Rothenberg ME. Eosinophilic esophagitis. $N$ Engl J Med. 2004;351(9):940-941.

6. Sgouros SN, Bergele C, Mantides A. Eosinophilic esophagitis in adults: a systematic review. Eur J Gastroenterol Hepatol. 2006;18(2):211-217.

7. Dellon ES, Gonsalves N, Hirano I, et al. ACG clinical guideline: Evidenced based approach to the diagnosis and management of esophageal eosinophilia and eosinophilic esophagitis (EoE). $\mathrm{Am} \mathrm{J}$ Gastroenterol. 2013;108(5):679-692.

8. Straumann A, Spichtin HP, Grize L, et al. Natural history of primary eosinophilic esophagitis: a follow-up of 30 adult patients for up to 11.5 years. Gastroenterology. 2003;125:1660-1669.

9. Kelly KJ, Lazenby AJ, Rowe PC, et al. Eosinophilic esophagitis attributed to gastroesophageal reflux: improvement with an amino acidbased formula. Gastroenterology. 1995;109(5):1503-1512.

10. Spergel JM, Andrews T, Brown-Whitehorn TF, et al.Treatment of eosinophilic esophagitis with specific food elimination diet directed by a combination of skin prick and patch tests. Ann Allergy Asthma Immunol. 2005;95(4):336-343.

11. Kagalwalla AF, Sentongo TA, Ritz S, et al. Effect of six-food elimination diet on clinical and histologic outcomes in eosinophilic esophagitis. Clin Gastroenterol Hepatol. 2006;4(9):1097-1102.

12. Molina-Infante J, Katzka DA, Gisbert JP. Review article: proton pump inhibitor therapy for suspected eosinophilic oesophagitis. Aliment Pharmacol Ther. 2013;37(12):1157-1164.

13. Remedios M, Campbell C, Jones DM, et al. Eosinophilic esophagitis in adults: clinical, endoscopic, histologic findings, and response to treatment with fluticasone propionate. Gastrointest Endosc. 2006;63(1):3-12.

14. Konikoff MR, Noel RJ, Blanchard C, et al. A randomized, doubleblind, placebo-controlled trial of fluticasone propionate for pediatric eosinophilic esophagitis. Gastroenterology. 2006;131(5):1381-1391.

15. Butz BK, Wen T, Gleich GJ, et al. Efficacy, dose reduction, and resistance to high-dose fluticasone in patients with eosinophilic esophagitis. Gastroenterology. 2014;147(2):324-333.

16. Schaefer ET, Fitzgerald JF, Molleston JP, et al. Comparison of oral prednisone and topical fluticasone in the treatment of eosinophilic esophagitis: a randomized trial in children. Clin Gastroenterol Hepatol. 2008;6(2):165-173. 
17. Mariusz Rosołowski, Maciej Kierzkiewicz .Etiology, diagnosis and treatment of infectious esophagitis. Prz Gastroenterol. 2013;8(6):333337.

18. Yang IA, Fong KM, Sim EH, et al.Inhaled corticosteroids for stable chronic obstructive pulmonary disease. Cochrane Database Syst Rev. 2012;18(2):CD002991.

19. Aun MV, Ribeiro MR, Costa Garcia CL, et al. Esophageal candidiasis-an adverse effect of inhaled corticosteroids therapy. J Asthma . 2009;46(4):399-401.

20. Lindberg GM, Van Eldik R, Saboorian MH. A case of herpes esophagitis after fluticasone propionate for eosinophilic esophagitis. Nat Clin Pract Gastroenterol Hepatol. 2008;5(9):527-530.
21. Patra S, Samal SC, Chacko A, et al.Cytomegalovirus infection of the human gastrointestinal tract. J Gastroenterol Hepatol. 1999;14(10):973976

22. Eymard D, Martin L, Doummar G, et al.Herpes simplex esophagitis in immunocompetent hosts. Can J Infect Dis. 1997;8(6):351-353.

23. Chatis $\mathrm{Pa}$, Miller $\mathrm{CH}$, Schrager Le, et al.Successful treatment with foscarnet of an acyclovir-resistant mucocutaneous infection with herpes simplex virus in a patient with acquired immunodeficiency syndrome. $N$ Engl J Med. 1989;320(5):297-300. 